\title{
Nasopharyngeal Carcinoma by AJCC v8 Stage
}

National Cancer Institute

\section{Source}

National Cancer Institute. Nasopharyngeal Carcinoma by A/CC v8 Stage. NCI Thesaurus.

Code C132816.

A term that refers to the staging of nasopharyngeal carcinoma according to the American Joint Committee on Cancer, 8th edition. 